\title{
Innovative Development of the Inspired Sinewave Device to Measure Lung Functions and Inhomogeneity for Diagnosis and Evaluations of Early Lung Diseases
}

\author{
P. Anh Phan ${ }^{1}$, H. Quoc Le ${ }^{2}$, C. Hieu Le ${ }^{3}$, D. Long Vo ${ }^{4}$, A. Tuan Mai ${ }^{5}$, N. Tam Le ${ }^{6}$, M. Hoa Le \\ ${ }^{1}$ The Nuffield Division of Anaesthetics, University of Oxford, Oxford, UK \\ ${ }^{2}$ Saigon Hi-Tech Park - SHTP, Ho Chi Minh, Vietnam \\ ${ }^{3}$ Faculty of Engineering and Science, University of Greenwich, Kent, United Kingdom \\ ${ }^{4}$ Ho Chi Minh City Medicine and Pharmacy University, Ho Chi Minh, Vietnam \\ ${ }^{5}$ Hanoi University of Science and Technology, Hanoi, Vietnam \\ ${ }^{6}$ IBM Cloud, IBM Vietnam, Hanoi, Vietnam \\ ${ }^{7}$ Northumbria University, Newcastle upon Tyne, United Kingdom
}

\begin{abstract}
Surprisingly, lung disease is still one of the leading causes of deaths in the developed countries, including UK. According to the UK National Health Service (NHS), Chronic Obstructive Pulmonary Disease (COPD) is the fifth biggest killer disease in the UK, killing approximately 25,000 people a year. This problem is even worse in developing countries such as Vietnam, India and China, where air pollution is a big problem and the disease awareness is under-recognised. The NHS has set out one of its challenges is to identify people with lung disease earlier in the disease's development pathway, in order to provide more effective and timely intervention and treatment. This paper presents a novel Inspired Sinewave Device (ISD) to measure lung function and inhomogeneity. Both set of information are important for diagnosis and detection of early lung diseases. ISD has the potential to replace or supplement the traditional spirometry in the routine lung function testing. The paper describes both the principle of ISD and a set of experimental results demonstrating the capability of ISD to asymptotically detect asthmatic symptoms. Finally the paper discusses the future plan, including the testing of 300+ COPD patients at the Oxford Respiratory Trials Unit in UK, and the potential collaborations among research institutions in $\mathrm{Vi}$ etnam and UK about cost-effective and innovative developments of smart devices, biosensors, lab-on-chips and telehealth solutions for the routine lung function testing, diagnosis and evaluations of early lung diseases.
\end{abstract}

Keywords - Lung Function Testing, Respiratory Disease, Asthma, Chronic Obstructive Pulmonary Disease, Medical Device

\section{INTRODUCTION}

According to the UK National Health Service (NHS), Chronic Obstructive Pulmonary Disease (COPD) is the fifth biggest killer disease in the UK, killing approximately 25,000 people a year [1]. An estimated 3 million people have COPD in the UK. Although for around 2 million of this group, their COPD remains undiagnosed (Healthcare Commission 2006) [2].

In the early stages of COPD, patients are largely free of symptoms but, as the disease progresses, patients report symptoms of breathlessness, coughing and chest tightness.
COPD primarily affects people aged over 45 and is characterised by a chronic, slowly progressive decline in lung function, usually associated with exposure to cigarette smoke, but occasionally with exposure to air pollution or other noxious particles or gases. The total annual cost of COPD to the NHS is estimated to be over $£ 800$ million for direct healthcare costs, which equates to $£ 1.3$ million per 100,000 people [2]. This problem is even worse in a developing country like Vietnam. With the population almost as twice as the UK, the problem is certainly bigger. Air pollution and smoking are the two biggest causes of COPD and are known problem in Vietnam. The numbers of smokers in developed countries have been steady or in a decline, whereas there is no sign of reducing in Vietnam. Moreover, due to limited resources of the healthcare system, the diagnosis, prevention, and treatment of the disease have not been addressed properly. Most people in Vietnam only go to hospitals when they are sick. At this time, COPD is already at a severe state and irreversible. There is a need for an accurate, affordable device acting as a screening test for lung function and disease, in order to identify people with disease early or encourage smoking cessation.

The Inspired Sinewave Device (ISD) is a novel device to measure lung functions and inhomogeneity. Both sets of information are important to the diagnosis and evaluations of early lung diseases. It works by delivering small doses of tracer gases into the patient's breaths and measuring the "response" in the expired breaths. By using an advanced model of the lung and circulatory system, the required parameters of the lung are estimated from the collected data. With this setup, the patients only require to breathe simultaneously through a mask. The technique does not rely on the patient's effort, which is known to be unreliable in the traditional spirometry. In a way, the ISD aims to bridge the gap between the insensitive and unreliable traditional spirometry and the accurate but expensive imaging techniques (such as CT or MRI), as an affordable first-point-of-care screening test of lung function and disease.

This paper describes the principle of the device, with more details having been given elsewhere [3-5]. This is followed by a presentation of two clinical studies demon- 
strating the capability of the device to measure functional residual capacity and lung inhomogeneity in a group of healthy and asymptotically asthmatic subjects. The paper then discusses the future plan of the project, including the testing of $300+$ COPD patients at the Oxford Respiratory Trials Unit in UK. Finally, we discuss about the potential collaborations among research institutions in Vietnam and UK about the cost-effective and innovative development of devices for the routine lung function testing for diagnosis and evaluations of early lung diseases.

\section{METHOD}

The method section describes the principle of the device and the setup of the 2 clinical studies.

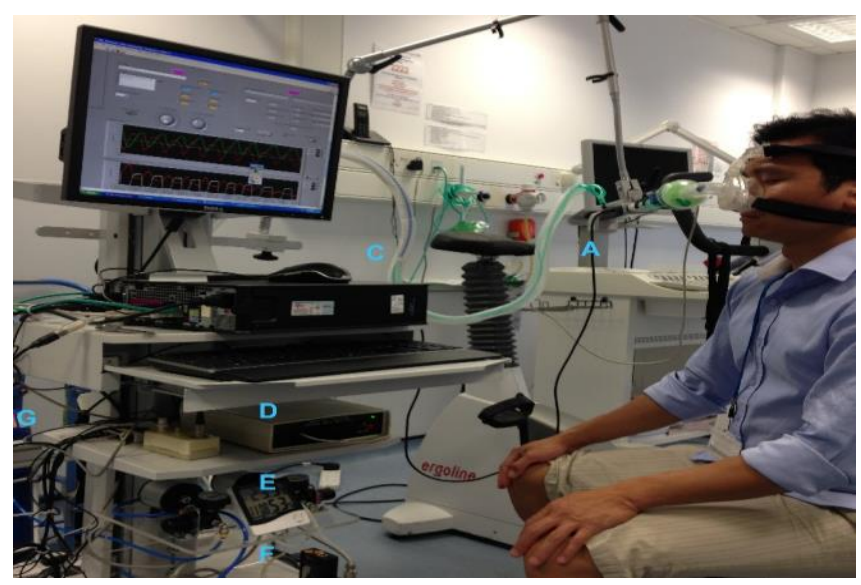

Fig.1 The hardware setup of the inspired sinewave device

\section{A. The principle of the Inspired Sinewave Device}

More details of the hardware and underlying mathematical equations have been described elsewhere [3-5]. We aim to give only a brief introduction here.

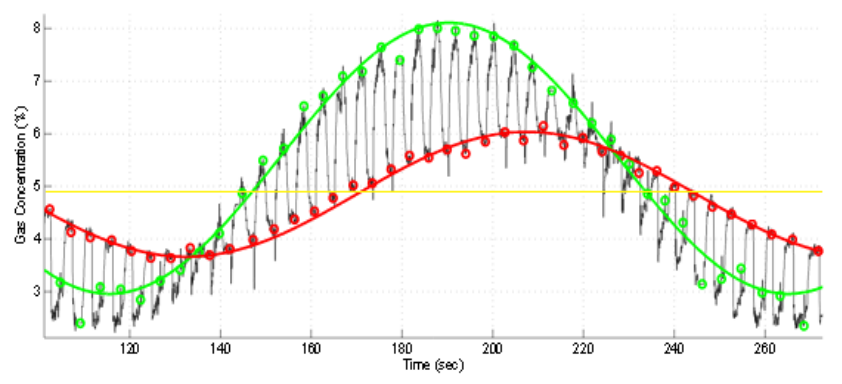

Fig. 2 Raw Data from the ISD in a healthy volunteer. Green circles and red circles are the inspired concentration and the expired concentration of each breath. Green trace and red trace are the inspired sinewave and expired sinewave respectively. Red trace: [N2O] in expired gas. Arrows mark change in sine wave amplitude between inspired and expired gas
The hardware setup of the current prototype of the device is given in Fig.1. The tracer gas, $\mathrm{N} 2 \mathrm{O}$ in this case, is delivered to the patient through a nasal mask. The control system decides the amount of $\mathrm{N} 2 \mathrm{O}$ based on the instantaneous flow of the breath. A $\mathrm{N} 2 \mathrm{O}$ gas sensor and a flow sensor are mounted on the breathing circuit to measure the flow and concentration in real time. The control algorithm is implemented in LabVIEW and the analysis and model are implemented in Matlab.

The mathematical principle of the device is as follows. By forcing the inspired concentrations of $\mathrm{O}_{2}$ and $\mathrm{N}_{2} \mathrm{O}$ to oscillate sinusoidally with very low amplitudes (3-5\%) and low mean for $\mathrm{N}_{2} \mathrm{O}(3 \%)$, the expired concentrations of $\mathrm{O}_{2}$ and $\mathrm{N}_{2} \mathrm{O}$ also follow a sinewave pattern (Fig. 2). Lung parameters then can be estimated from the responding amplitudes and phases of the expired concentrations. The larger the lung parameters, the greater are the attenuations of the expired oscillations:

$$
\frac{\Delta P_{E}}{\Delta P_{I}}=\frac{1}{\sqrt{\left(1+\frac{\lambda_{N_{2} O} \dot{Q}_{P}}{\dot{V}_{A}}\right)^{2}+\left(\frac{2 \pi}{T}\right)^{2}\left(\frac{V_{A}}{\dot{V}_{A}}\right)^{2}}}
$$

in which:

$\Delta P_{E}:$ the magnitude of the expired sinewave

$\Delta P_{I}$ : the magnitude of the inspired sinewave

$\lambda_{N_{2} \mathrm{O}}$ : the solubility of nitrous oxide in the blood

$\dot{Q}_{P}$ : the pulmonary blood flow ( $\left.\mathrm{L} / \mathrm{min}\right)$

$\dot{V}_{A}$ : the volume of gas ventilated by the lung (L/min)

$T:$ the sinewave period applied by the technique (mins)

$V_{A}$ : the lung volume (L)

Based on eq (1), $V_{A}$ and $\dot{Q}_{P}$ can be estimated by measuring $\Delta P_{E} / \Delta P_{I}$ at 2 or more periods. Interested readers can refer to more detailed publications [6], [7] for full mathematical description of the technique.

\section{B. The setup of the 2 clinical studies}

\section{a) Clinical study I: Comparison with body plethysmography}

The aim of this study is to compare the functional residual capacity (FRC) measured by the ISD and body plethysmography. Body plethysmography FRC was taken with a MasterScreen Body (Cardinal Health) body plethysmograph, located in the Lung Function Laboratory, Churchill Hospital, Oxford UK. 32 healthy subjects with no history of cardiopulmonary diseases volunteered to participate in this study. Of which, 12 were female and 20 were male, 5 were active smokers. Unless otherwise specified, participants 
were taught to breathe in a relaxed way through airtight, surgical masks connected to the device. Participants were also reminded to keep their mouths sealed to prevent incidences of gas leakage and were asked to relax in the ten minutes before measurement runs. Time period of the input tracer sinewave was fixed at 180 seconds ( 3 minutes) for the duration of this study.

The sinewave period of 3 minutes were chosen through extensive empirical test and simulation study. It was found that at 3-min sinwave, the estimated lung parameters were closest to the clinically meaningful values. The dependency of the estimated parameters on the period of the applied sinewave was actually an advantage and was used to investigate the lung inhomogeneity in the Clinical Study II below.

\section{b) Clinical study II: Period response for investigation of lung inhomogeneity}

The aim of this study was to determine whether the time period response of a subjects' lungs may also indicate the presence of ventilation inhomogeneity, which whilst more pronounced in some respiratory diseases e.g. asthma[8], may still exist to some degree in healthy subjects [9]. Seventeen healthy volunteers (11 male/6 female) were recruited. Six measurements of normal breathing into the ISD were made on each volunteer over a two hour period; the period of the applied sinewave was set at: $0.51,2,3,4$ and 5 mins, for each of the measurements respectively although the order in which they were performed was randomised.

\section{RESUlts}

\section{A. Clinical study I: comparison study with body plethysmography}

The comparison was carried out in the form of a Bland Altman plot [10], and the result was shown in Fig. 3. The bias was estimated to be $-0.68 \mathrm{~L}$, and the limits of agreement were $0.14 \mathrm{~L}$ to $-1.50 \mathrm{~L}$. The BIAS $\pm \mathrm{SD}$ of $-0.68 \pm 0.42 \mathrm{~L}$ showed that the ISD had similar precision to other techniques when comparing with the body plethysmography (BP) in the literature [11, 12], demonstrating the capability of the ISD in measuring functional residual capacity.

\section{B. Clinical study II: Period response for investigating of lung inhomogeneity}

The result was displayed in the form of the ratio between the FRC measured by the ISD and the FRC measured by body plethysmography. Figure 4 shows the mean of the ratio over all subjects across the tested periods. It could be seen that the ratio in older asymptotic asthmatic subjects was both smaller and steeper as the period changed from 4 mins to 0.5 mins. A smaller ratio suggested that "gas trap- ping" existed in the lung. A steeper change of the ratio as the period changed suggested lung inhomogeneity. We therefore suggested the use of this ratio as an index to describe the level of lung inhomogeneity, with more details given in the our previous patent application [3].

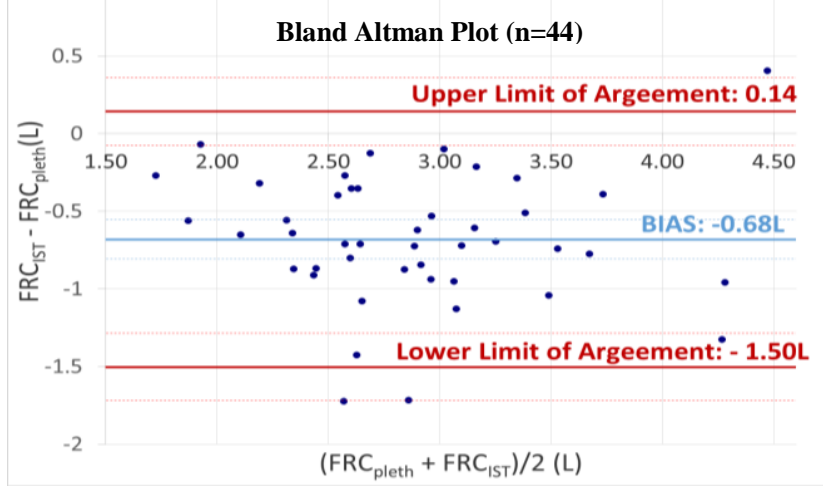

Fig.3 Bland Altman plot comparing FRC measured by the ISD and body plethysmography. Solid blue and red lines represent the bias and the limits of agreement respectively. Dashed lines show the $95 \%$ confidence intervals $\mathrm{f}$ these estimates.

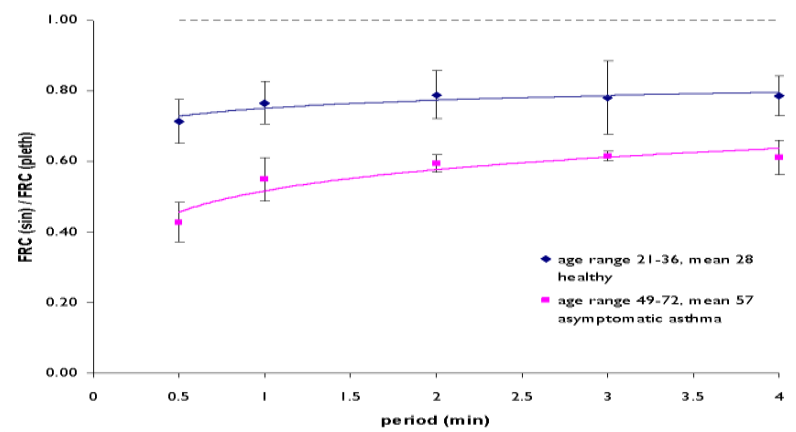

Fig. 4 The period response of the ratio between the FRC measured by the ISD and the body plethysmography, in 2 patient groups. We proposed to use this ratio as an index to investigate lung inhomogeneity.

\section{DisCUSSION AND CONCLUSION}

On the back of the preliminary results, including the studies in this paper, we have secured a $£ 0.7 \mathrm{mil}$ from the National Institute of Health Research Invention for Innovation grant (UK), to further test the device for 2 applications: the continuous monitoring of lung function and blood flow of patients in operating theatres and intensive care units, and the lung function testing and diagnosis of outpatients with COPD. Our coming plan is to test the ISD on over 300 patients with COPD over the next 2 years, at the Churchill hospital, Oxford, UK. The main aim of this study is to map the measurements obtained from the ISD with the assessments from current standard COPD care, to validate whether the ISD is capable of detecting different levels of disease severity. The secondary aim of the study is to follow up 
these patients over 3-5 years, to validate whether the ISD has a better predictive probability of disease deterioration over current standard tests. If this hypothesis is validated, the ISD will be a useful diagnostic tool to determine which patients should require further testing and earlier intervention.

The developed ISD is able to address the lung function testing and disease diagnosis, particularly the chronic obstructive pulmonary disease; it has potential impact in both developed and developing countries, including UK and Vietnam. Especially, Vietnam has a large population of 90 millions. According to the World Economic Forum, Viet Nam is one of the ten countries with the worst air pollution among 132 countries whose environments were surveyed in relation to effects on human health. Also, according to the WHO, Viet Nam was among countries with the highest mortality rates due to ambient air pollution, 200-230 cases per million people per year. Moreover, in the low-income countries, the poor quality of diagnosis, detection of early diseases and cancer scanning leads to the survival rates of cancers and successful treatments of diseases are also much lower. By 2020, there will be between 15 and 17 million new cases of cancer every year, $60-70 \%$ new cases of cancer and nearly $70 \%$ of cancer deaths will be in economically disadvantaged countries [14].

Followed the successful preliminary studies, the present and ongoing plan is to further validate the device in a larger group of COPD in the UK. This opens potential collaborations among scientists in Vietnam and UK in both the technical development and the clinical trial of the device in hospital settings. Concretely, the following are the preliminary plan for RTD collaborations: (1) Technical collaboration: to assist in developing, prototyping the hardware and instrumentation of components of the device, such as the sensing system; and (2) Clinical trial collaboration: to perform the trial of the device in hospital settings, exploring a new patient demographics, totally different from the UK patients in term of size, races, living conditions etc. Costeffective solutions for design and manufacture of the devices should be the core objective. In addition, with the recent advancements in micro \&nano manufacturing, smart biosensors and lab-on-chips can be developed for early detection of diseases and cancer screening. A research group at Hanoi University of Science and Technology has been working on developments of biosensors for characterization of cancer cells, early detections of diseases and cancer screening. These could be additional functions of ISD for effective diagnosis and evaluations of early lung diseases.

A successful collaboration in Research \& Technology Development (RTD) would strengthen the link between research institutions in Vietnam and UK, leading to the fruitful research results, and enhancements of human resources developments in Biomedical Engineering for Vietnam. Also, the successful developments of ISD and collaborations between Vietnam and UK will have more potential impacts on a global scale, in both developed and developing countries, especially RTD collaborations about the cost-effective and innovative developments of ISD, biosensors, lab-on-chips, and telehealth solutions for the routine lung function testing, diagnosis and evaluations of early lung diseases.

\section{ACKNOWLEDGMENT}

The studies presented in this paper were funded by the Engineering and Physical Science Research Council, UK. The ongoing research is being funded by the National Institute of Health Research, through an Invention for Innovation grant, UK. British Council - Newton Fund is acknowledged for their support.

\section{REFERENCES}

1 NHS England» 6. Respiratory disease: www.england.nhs.uk [Accessed: April 2016]

2 NHS Chronic Obstructive Pulmonary Disease Costing Report (2011): www.nice.org.uk [Accessed: April 2016]

3 P. A. Phan et al (2015) Method and Apparatus for Measurement of Cardiopulmonary Function. Patent Pub. No: O/2015/145115

4 L. Clifton et al. (2012) A novel non-invasive method for estimating cardiopulmonary variables using breath-by-breath tracer gas injection'. Cardiovascular Devices And Systems;1: 1900108

5 L. Clifton et al. (2013) Assessment of Lung Function using a Noninvasive Oscillating Gas-forcing Technique. Respiratory Physiology \& Neurobiology; 189 (1): 174-182

6 E. M. Williams et al. (1998) Pulmonary blood flow measured by inspiratory inert gas concentration forcing oscillations', Respir. Physiology; 113 (1): 47-56

7 L. Clifton et al. (2013) Assessment of lung function using a noninvasive oscillating gas-forcing technique. Respir. Physiol. Neurobiology; 189 (1): 174-182

8 C. C. B. Almeida et al. (2011) Volumetric capnography to detect ventilation inhomogeneity in children and adolescents with controlled persistent asthma. J. Pediatr. (Rio J.); 87 (2): 163-168.

9 S. Verbanck et al. (1997) Ventilation distribution during histamine provocation. J. Appl. Physiology; 83(1)6: 1907-1916.

10 J. M. Bland and D. G. Altman (1999) Measuring agreement in method comparison studies', Stat. Methods Med. Res.; 8(2): 135-160

11 I. J. Cliff et al. (1999) Comparison of two new methods for the measurement of lung volumes with two standard methods'. Thorax; 54(4): 329-333

12 C. R. O'Donnell et al. (2010) Comparison of plethysmographic and helium dilution lung volumes: which is best for COPD?. Chest; 137(5): 1108-1115

13 L. Brewer and J. Orr (2009) Measurement of Functional Residual Capacity of the Lung by Nitrogen Washout, Carbon Dioxide Rebreathing and Body Plethysmography in Healthy Volunteers. Utah Space Grant Consortium: http://digitalcommons.usu.edu [Accessed: April 2016]

14. Miriam L.G. et al. (2013) Cancer in developing countries: The next most preventable pandemic. The Global problem of cancer. Critical Reviews in Oncology/Hematology; 88:117-122.

Author: Phi Anh Phan

Institute: University of Oxford

Address: Level 6, West Wing, John Radcliffe Hospital

City: Oxford

Country: UK

Email: phi.phan@nden.ox.ac.uk 